\title{
Attachment of Nucleosides to Solid-Phase Supports
}

The first step in any solid-phase synthesis is the covalent attachment of a monomeric unit (i.e., a nucleoside or mononucleotide) to the surface of an insoluble support. For oligonucleotide synthesis, this process is performed separately from the automated coupling steps used for chain elongation because of the different coupling chemistry involved. This unit provides protocols for using amide and ester linkages to attach nucleosides to long-chain alkylamine-controlled-pore glass (LCAA-CPG) bead supports. Protocols and descriptions of the coupling chemistry used by automated synthesizers to create internucleotide phosphodiester linkages are contained in subsequent units, and the properties and applications of a large variety of solid supports are reviewed in UNIT 3.1.

Although prederivatized solid supports are commercially available from a number of sources, preparation of these materials in the lab is a very reasonable alternative to their purchase: the procedures are very simple, and a single support synthesis can produce sufficient material for a great number of oligonucleotide syntheses. Significant savings can also be obtained by filling one's own synthesis columns instead of purchasing ready-to-use columns. In addition, many minor-nucleoside- and special non-nucleosidederivatized supports are either not commercially available or quite costly.

This unit provides protocols for attaching nucleosides to supports via two different dicarboxylic acid linkers: succinic anhydride, $\mathbf{S . 4}$, and hydroquinone- $O, O^{\prime}$-diacetic acid (HQDA or Q-linker), S.8. Basic Protocol 1 describes how commercially available nucleoside-3'-O-succinate hemiesters can be attached to LCAA-CPG through an amide linkage. Alternate Protocol 1 describes how nucleosides with free $3^{\prime}-\mathrm{OH}$ groups can be attached to succinylated LCAA-CPG through an ester linkage. Basic Protocol 2 describes how to synthesize nucleoside- $3^{\prime}-O$-hydroquinone diacetyl hemiesters from nucleosides and HQDA and how they are coupled to LCAA-CPG. Alternate Protocol 2 describes the preparation of an HQDA-derivatized LCAA-CPG support and how nucleosides with free $3^{\prime}-\mathrm{OH}$ groups can be coupled to it through an ester linkage. Protocols for quantitating dimethoxytrityl (DMTr) groups attached to a support (Support Protocol 1), for converting surface amino or hydroxyl groups into DMTr derivatives for quantitation (Support Protocol 2), and for quantitating surface carboxylic acid groups as either $p$-nitrophenol (Support Protocol 3) or $\mathrm{N}$-monomethoxytrityl-6-amino-1-hexanol (Support Protocol 4) derivatives are also included.

The succinic acid linker has the advantage of being the most widely used linker arm, and both succinic anhydride and protected nucleoside-3'-O-succinyl hemiester starting materials are readily available. On the other hand, the hydroquinone- $O, O^{\prime}$-diacetic acid linker offers greater compatibility with base-sensitive sequence modifications and greater synthetic throughput, because it can be cleaved under milder and faster conditions than can the succinyl linker. No changes in oligonucleotide synthesis protocols, other than reducing the cleavage time (i.e., from 60 to $90 \mathrm{~min}$ to only 2 to $3 \mathrm{~min}$ ), are required when Q-linker is substituted for a succinyl linker.

Almost any application requiring synthetic oligonucleotides can be satisfied using one of these linker arms. The major exceptions are applications that require leaving the final oligonucleotide immobilized on the support after synthesis and deprotection, i.e., the direct synthesis of immobilized oligonucleotide libraries. Researchers interested in this application should consult UNIT 3.1 for the appropriate noncleavable linker arms. 


\section{STRATEGIC PLANNING: SELECTING A LINKER ARM AND COUPLING PROTOCOL}

The protocols in this unit involving the two linker arms (succinic acid and HQDA) are each divided into two separate procedures, depending on the site where the linker is first attached. When the linker arm is attached to the $3^{\prime}$-hydroxyl position of a suitably protected nucleoside to produce a nucleoside-3'-O-hemiester, such as $\mathbf{S . 2}$ or $\mathbf{S . 9}$, a separate synthetic preparation is necessary for each different nucleoside. The alternate approach, which first couples the linker arm to the support to give a carboxyl-derivatized support, such as $\mathbf{S . 5}$ or $\mathbf{S . 1 2}$, eliminates the need for a separate hemiester preparation for each nucleoside. The coupling reaction between a carboxyl-derivatized support and the secondary $3^{\prime}-\mathrm{OH}$ of a nucleoside (i.e., S.6), however, is more difficult; this method is also slower and generates less nucleoside attached to the surface of the support.

The selection of the best protocol depends on the cost and availability of the required nucleosides as well as each researcher's experience with organic synthesis. Researchers who want to perform only minimal synthetic manipulations should choose Basic Protocol 1 (Fig. 3.2.1). This method consists of only one coupling step, if commercially available nucleoside succinate, $\mathbf{S . 2}$, is purchased. Researchers requiring supports derivatized with minor or unusual nucleosides, which are often very costly, and who will be preparing oligonucleotides with no unusual sensitivity to basic cleavage conditions should use Alternate Protocol 1, involving succinylated LCAA-CPG (Fig. 3.2.2). The succinylated LCAA-CPG support, $\mathbf{S . 5}$, is very easy to prepare, and this approach minimizes consumption of rare and expensive nucleosides.

The two protocols using the Q-linker require a slightly greater familiarity with synthesis methodology, although these protocols are still within the ability of most "beginners." Basic Protocol 2 describes the preparation of nucleoside hemiesters of HQDA (which are not yet commercially available) and their subsequent attachment to LCAA-CPG (Fig. 3.2.3). This method has the advantage of providing higher nucleoside loadings and is particularly important for synthesis on reusable supports (Pon et al., 1998, 1999). Researchers with more limited chemical resources should use Alternate Protocol 2 to prepare HQDA-derivatized LCAA-CPG (Fig. 3.2.4), at least until nucleoside-3'-O-hemiesters of HQDA become commercially available. As with Alternate Protocol 1 for the succinyl linker, this method is simple and eliminates the need for nucleoside- $3^{\prime}-O$-hemiesters of HQDA, S.9. This method is also attractive because it avoids any use of pyridine. Use of the Q-linker rather than the succinyl linker is highly recommended for facilities wishing to increase their daily synthesis throughput or for projects requiring base-sensitive sequences, such as methyl phosphonate or methyl phosphotriester backbones, basesensitive nucleosides, and 3'-linked fluorescent dye labels.

BASIC PROTOCOL 1

Attachment of Nucleosides to Solid-Phase Supports

\section{COUPLING OF NUCLEOSIDE-3'-O-SUCCINYL HEMIESTERS TO LCAA-CPG SUPPORT}

This protocol (Fig. 3.2.1) attaches $5^{\prime}$-dimethoxytrityl-N-protected nucleoside-3'-O-succinate hemiesters, S.2, directly to long-chain alkylamine-controlled-pore glass (LCAACPG; S.1). This protocol has the advantage of using commercially available succinate

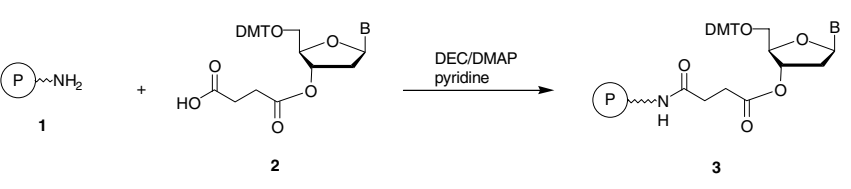

Figure 3.2.1 Coupling of LCAA-CPG, S.1, directly to a nucleoside-3'-O-succinyl hemiester, S.2, as described in Basic Protocol 1. DMT, dimethoxytrityl group. 


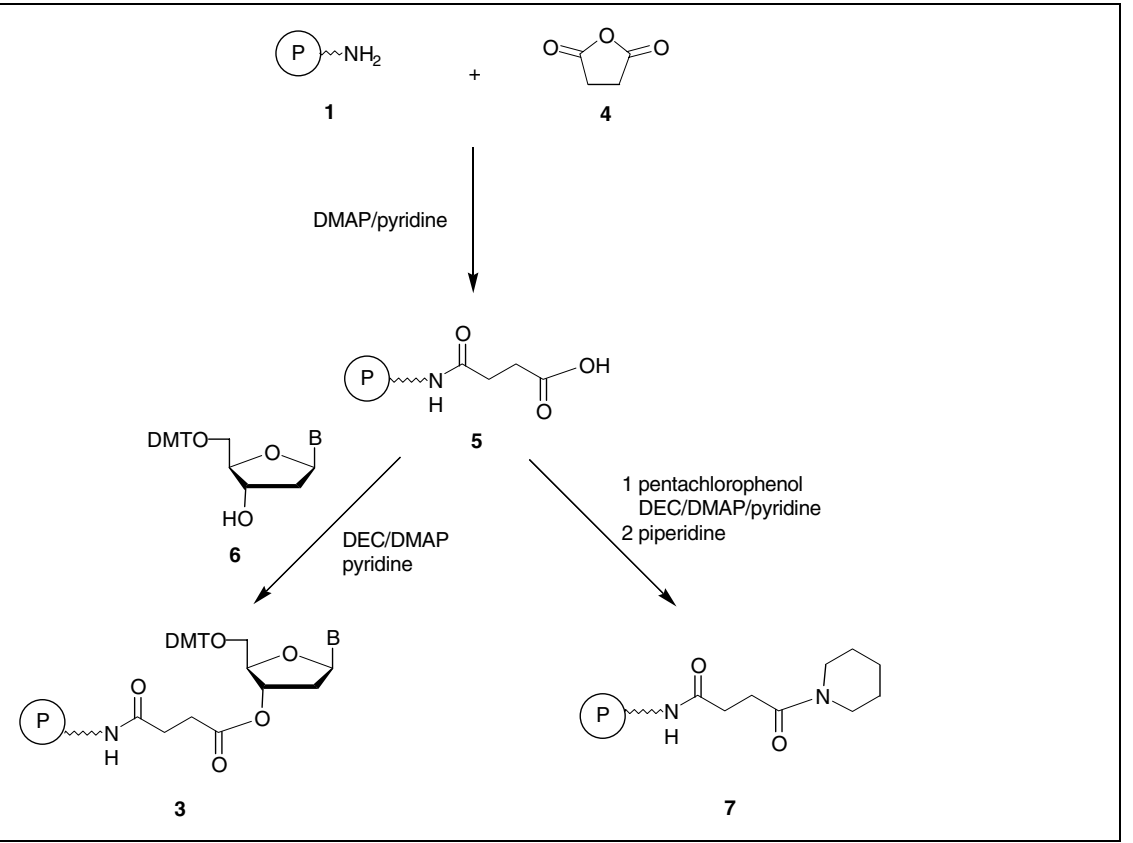

Figure 3.2.2 Synthesis of succinylated support, S.5, by reaction of succinic anhydride, S.4, with LCAA-CPG, S.1. Nucleosides with a free $3^{\prime}-\mathrm{OH}$ group, S.6, can then be esterified to $\mathbf{S} .5$ to yield support S.3. Unreacted succinic acid groups are blocked by conversion into an active pentachlorophenyl ester followed by treatment with piperidine to yield the unreactive site shown as support S.7. DMT, dimethoxytrityl group.

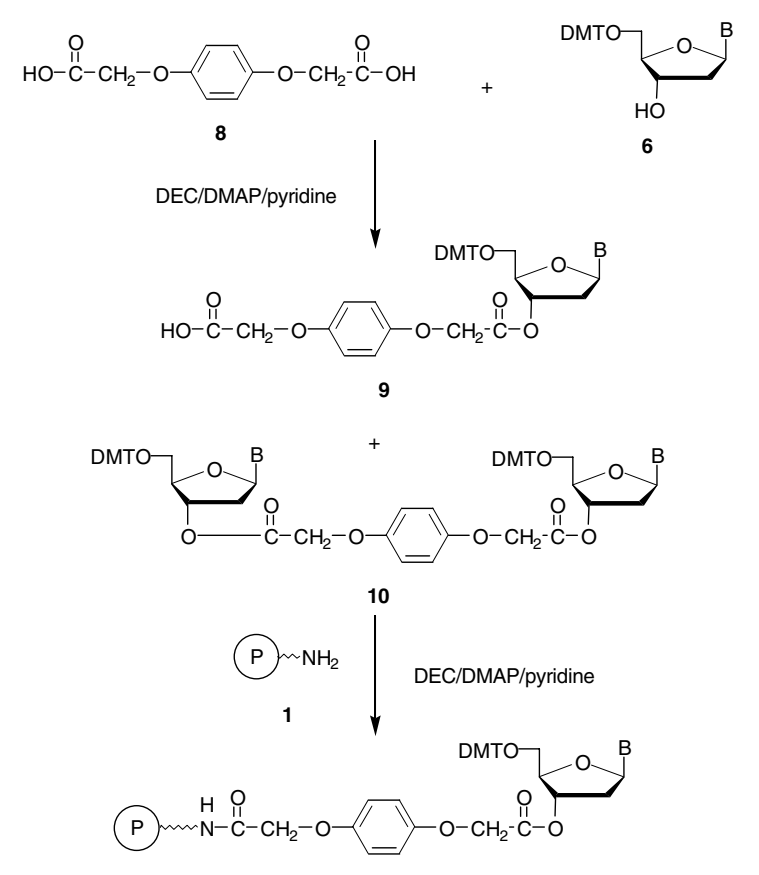

11

Figure 3.2.3 Synthesis of nucleoside-3'-O-hydroquinone diacetyl hemiester, S.9, from HQDA, S.8, and a protected nucleoside, S.6. A nucleoside diester, S.10, is also produced as a minor byproduct. The mixture of $\mathbf{S . 9}$ and $\mathbf{S . 1 0}$, however, is used directly in the coupling reaction with LCAA-CPG, S.1, to produce nucleoside-derivatized support S.11. DMT, dimethoxytrityl group.

Synthesis of Unmodified Oligonucleotides 


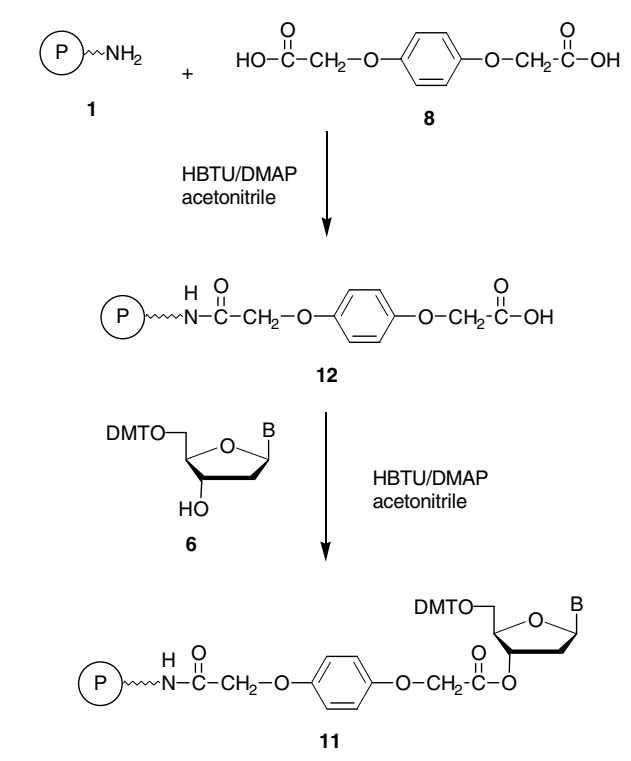

Figure 3.2.4 Coupling of LCAA-CPG, S.1, to HQDA, S.8, using HBTU and DMAP to produce HQDA-derivatized support S.12. A suitably protected nucleoside, S.6, with a $3^{\prime}-\mathrm{OH}$ group is then esterified to $\mathbf{S . 1 2}$ to generate nucleoside-derivatized support S.11. DMT, dimethoxytrityl group.

hemiester starting materials, S.2, and so the synthesis of these reagents is not included in this protocol. Researchers requiring derivatives of nucleosides other than the four common deoxyribonucleosides ( $\mathrm{dA}, \mathrm{dC}, \mathrm{dG}$, and $\mathrm{T})$ may need to prepare them by coupling succinic anhydride with the appropriately protected rare nucleoside, according to established procedures (Gait et al., 1980; Miyoshi et al., 1980; Chow et al., 1981; Kumar et al., 1993; Markiewicz et al., 1997).

\section{Materials}

LCAA-CPG beads (other amino-derivatized nonswelling support materials may also be used; see recipe)

$5^{\prime}$-Dimethoxytrityl-N-protected nucleoside $3^{\prime}$-O $O$-succinyl hemiester (see recipe)

4-Dimethylaminopyridine (DMAP)

1-(3-Dimethylaminopropyl)-3-ethylcarbodiimide hydrochloride (DEC; see recipe)

Triethylamine, reagent grade

Anhydrous pyridine, for coupling reactions (see recipe)

Methanol, reagent grade

Dichloromethane, reagent grade

Pyridine, reagent-grade, for washing supports

Cap A capping solution (see recipe)

Cap B capping solution (see recipe)

Screw-capped glass vials or round-bottom flasks

Pasteur pipet

Buchner funnel

Whatman no. 1 filter paper

Shaker, wrist action or similar

Attachment of

Additional reagents and solutions for trityl analysis (see Support Protocol 1) 
CAUTION: Carbodiimide reagents, such as DEC, can cause severe skin or eye irritation and allergic reactions. Use appropriate skin and eye protection, and wipe up all work areas and utensils with a wet cloth immediately after use (DEC is water soluble).

NOTE: If the amino group loading of the starting support is not known, it is recommended that dimethoxyl derivatization to assay for amino and hydroxyl groups (see Support Protocol 2) be performed to verify the support's capacity before starting the nucleoside derivatization.

\section{Couple nucleoside $3^{\prime}$-O-succinyl hemiesters to $L C A A-C P G$}

1. Combine in a small glass screw-capped vial or round-bottom flask:

\section{$1.0 \mathrm{~g} \mathrm{LCAA-CPG}$}

0.2 mmol 5'-dimethoxytrityl-N-protected nucleoside $3^{\prime}$ - $O$-succinyl hemiester $12 \mathrm{mg}(0.1 \mathrm{mmol})$ DMAP

$382 \mathrm{mg}(2.0 \mathrm{mmol})$ DEC.

2. Add $80 \mu \mathrm{L}$ triethylamine and $\sim 5$ to $10 \mathrm{~mL}$ anhydrous pyridine. Quickly seal the vial, and shake at room temperature.

The reaction time used depends on the nucleoside loading desired. Reactions as short as $1 \mathrm{hr}$ can yield loadings of 20 to $30 \mu \mathrm{mol} / \mathrm{g}$, whereas overnight reactions can yield loadings of up to $60 \mu \mathrm{mol} / \mathrm{g}$. A coupling time of 2 to $3 \mathrm{hr}$, which usually gives a loading of between 30 and $40 \mu \mathrm{mol} / \mathrm{g}$, is generally most satisfactory.

3. If desired, periodically monitor the course of the coupling reaction by removing a small sample of support (10 to $20 \mathrm{mg}$ ) with a Pasteur pipet and applying it to a Buchner funnel. Wash with $20 \mathrm{~mL}$ methanol and then $20 \mathrm{~mL}$ dichloromethane; dry uncovered at room temperature, $\sim 15 \mathrm{~min}$. Quickly determine the nucleoside loading by trityl analysis (see Support Protocol 1).

4. After sufficient nucleoside loading has been obtained, filter off the support, working in the fume hood.

5. Wash successively with $\sim 5$ to $10 \mathrm{~mL}$ reagent-grade pyridine, $\sim 10$ to $20 \mathrm{~mL}$ methanol, and finally $\sim 20$ to $30 \mathrm{~mL}$ dichloromethane. Allow the support to dry at room temperature.

\section{Cap unreacted amino groups}

6 . Return the support to a clean vial or flask, and add equal volumes $(\sim 5 \mathrm{~mL})$ of Cap A and Cap B capping reagents. Seal the vial and shake 1 to $2 \mathrm{hr}$ at room temperature.

7. Filter off the support, and wash with $\sim 10$ to $20 \mathrm{~mL}$ methanol and then $\sim 20$ to $30 \mathrm{~mL}$ dichloromethane. Allow to air dry.

8. Determine the nucleoside loading by trityl analysis of a small portion of the final product (see Support Protocol 1).

Store at room temperature in a sealed screw-capped vial. Supports can be stored for several years at room temperature. 
This protocol describes the coupling of succinic anhydride, S.4, to LCAA-CPG, S.1, to produce a succinylated support, S.5 (Fig. 3.2.2). Suitably protected nucleosides, S.6, with a free $3^{\prime}-\mathrm{OH}$ group are then esterified to the succinylated support. Once the succinylated support has been prepared, only $5^{\prime}$-dimethoxytrityl-N-protected nucleosides (with free $3^{\prime}-\mathrm{OH}$ groups) are required. This provides an advantage over Basic Protocol 1, because a greater variety of nucleosides are commercially available as $5^{\prime}$ - and $\mathrm{N}$-protected derivatives than as $5^{\prime}$ - and $\mathrm{N}$-protected $3^{\prime}$ - $O$-succinates.

\section{Additional Materials (also see Basic Protocol 1)}

Succinic anhydride, reagent grade

Ninhydrin solution: $2 \%(\mathrm{w} / \mathrm{v})$ ninhydrin in ethanol

Pentachlorophenol, reagent grade

Piperidine, reagent grade

Water aspirator

$13 \times 75-$ mm glass test tube

Additional reagents and solutions for quantitative nitrophenol assay (see Support Protocol 3), $N$-monomethoxytrityl-6-amino-1-hexanol assay (see Support Protocol 4), and trityl analysis (see Support Protocol 1)

CAUTION: Carbodiimide reagents, such as DEC, can cause severe skin or eye irritation and allergic reactions. Use appropriate skin and eye protection, and wipe up all work areas and utensils with a wet cloth immediately after use (DEC is water soluble).

CAUTION: Pentachlorophenol is highly toxic.

CAUTION: Piperidine has a strong unpleasant odor and is highly toxic. Wear gloves and always handle in a fume hood.

CAUTION: Avoid skin contact with the ninhydrin solution; blue stains will form.

\section{Couple succinic anhydride to LCAA-CPG}

1. Combine in a screw-capped vial (such as a glass scintillation vial) or small roundbottom flask:

\section{$1.0 \mathrm{~g}$ LCAA-CPG}

$200 \mathrm{mg}(2.0 \mathrm{mmol})$ succinic anhydride

$24 \mathrm{mg}$ (0.2 mmol) DMAP.

Immediately add $\sim 5$ to $10 \mathrm{~mL}$ anhydrous pyridine and seal the vial.

Perform the pyridine addition quickly, and immediately seal the vial tightly, to minimize exposure to moisture in the air. Laboratories with very high humidity can avoid moisture contamination by sealing the vial with a septum and using a syringe for the pyridine transfer, but this is not necessary in most (air-conditioned) laboratories.

2. Shake the vial at room temperature overnight, using a speed sufficient to keep the support in a gentle suspension.

Do not use a magnetic stirrer for this or any subsequent steps, because the stir bar can break up the LCAA-CPG particles and produce unwanted fines.

Attachment of Nucleosides to Solid-Phase Supports

3. Inside a fume hood, filter off the LCAA-CPG using a Buchner funnel, Whatman no. 1 filter paper, and vacuum provided by a water aspirator. 
4. Wash the support successively with $\sim 5$ to $10 \mathrm{~mL}$ pyridine, then $\sim 10$ to $20 \mathrm{~mL}$ methanol, and finally $\sim 20$ to $30 \mathrm{~mL}$ dichloromethane.

5. Transfer the support to a piece of smooth, white paper and allow to sit uncovered at room temperature until dry, $\sim 15$ to $30 \mathrm{~min}$.

The succinylated LCAA-CPG support can be stored indefinitely at room temperature.

6. Place 2 to $3 \mathrm{mg}$ succinylated LCAA-CPG in a clean $13 \times 75-\mathrm{mm}$ glass test tube, and add 1 or 2 drops of ninhydrin solution. Warm the mixture until the solvent evaporates and the beads are dry.

Any unreacted amino groups will form a very dark blue color on the beads, whereas the succinylated support should only have a faint blue color. This is a qualitative test, and it is helpful to treat a sample of LCAA-CPG alongside the test material to observe the color difference.

7. Verify the extent of the succinylation reaction by performing the quantitative nitrophenol (see Support Protocol 3) or $N$-monomethoxytrityl-6-amino-1-hexanol (see Support Protocol 4) assay for carboxylic acid loading. If the test results are unsatisfactory, repeat the succinylation reaction on the support.

The carboxyl loading obtained on succinyl-derivatized LCAA-CPG should be close to the amino loading determined by the LCAA-CPG manufacturer. Owing to differences in the nature of the amino and carboxyl determinations, it is normal to observe some difference.

\section{Couple nucleoside to the succinylated LCAA-CPG}

8. Combine in a small screw-capped glass vial or round-bottom flask:

\section{0 g succinylated LCAA-CPG \\ 0.1 mmol 5'-dimethoxytrityl-N-protected 2'-deoxyribonucleoside $12 \mathrm{mg}(0.1 \mathrm{mmol})$ DMAP \\ $192 \mathrm{mg}(1.0 \mathrm{mmol})$ DEC.}

Add $80 \mu \mathrm{L}$ triethylamine and $\sim 5$ to $10 \mathrm{~mL}$ anhydrous pyridine. Quickly seal the vial, and shake overnight at room temperature.

9. Verify the progress of the coupling reaction by removing $\sim 10$ to $20 \mathrm{mg}$ of support using a Pasteur pipet. Apply the sample to a Buchner funnel, and wash with 10 to $20 \mathrm{~mL}$ methanol and then 20 to $30 \mathrm{~mL}$ dichloromethane. Allow the support to dry, and then determine the nucleoside loading by trityl analysis (see Support Protocol 1). If the loading is satisfactory (i.e., a minimum of 20 and preferably 30 to 40 $\mu \mathrm{mol} / \mathrm{g}$ ), then proceed to step 10 . Otherwise, extend the shaking for another day or repeat the reaction by adding more of the reagents (nucleoside, DEC, DMAP, and triethylamine) to the flask.

\section{Cap unreacted carboxyl and amino sites on the support}

10. Add $134 \mathrm{mg}(0.5 \mathrm{mmol})$ pentachlorophenol to the reaction mixture, and shake 16 to $24 \mathrm{hr}$ at room temperature.

Pentachlorophenol is added to convert any unreacted carboxyl groups into activated pentachlorophenyl esters. When treated with the cyclic amine piperidine (step 11), the pentachlorophenyl esters are converted into an unreactive amide, S.7.

11. Add $5 \mathrm{~mL}$ piperidine and continue shake $5 \mathrm{~min}$ at room temperature.

Prolonged exposure to piperidine will also hydrolyze the succinyl linker and decrease the nucleoside loading on the support. 
12. Inside a fume hood, filter off the LCAA-CPG beads and wash successively with $\sim 5$ to $10 \mathrm{~mL}$ reagent-grade pyridine, $\sim 10$ to $20 \mathrm{~mL}$ methanol, and finally $\sim 20$ to $30 \mathrm{~mL}$ dichloromethane. Allow the support to sit at room temperature until dry.

13. Return the support to a clean glass screw-capped vial or small round-bottom flask and add equal amounts $(\sim 5 \mathrm{~mL})$ of Cap A and Cap B capping reagents. Seal the vial and shake 1 to $2 \mathrm{hr}$ at room temperature.

This reaction is performed to acetylate any amino groups on the support that may not have been succinylated in the first reaction.

14. Filter off the LCAA-CPG on a Buchner funnel and wash with 10 to $20 \mathrm{~mL}$ methanol and then $\sim 20$ to $30 \mathrm{~mL}$ dichloromethane. Allow to dry at room temperature.

15. Determine the nucleoside loading by trityl analysis of a small portion of the final product (see Support Protocol 1).

Store at room temperature in a sealed screw-capped vial. Supports can be stored for several years.

BASIC PROTOCOL 2

Attachment of Nucleosides to Solid-Phase Supports

\section{COUPLING OF NUCLEOSIDE-3'- $O$-HYDROQUINONE- $O, O^{\prime}$-DIACETYL HEMIESTERS TO LCAA-CPG}

In this protocol, the hydroquinone- $O, O^{\prime}$-diacetic acid (HQDA), S.8, is first attached to the $3^{\prime}-\mathrm{OH}$ of a suitably protected nucleoside, S.6, by using 1-(3-dimethylaminopropyl)3 -ethylcarbodiimide hydrochloride (DEC) as the coupling reagent. Unfortunately, selective coupling to only one end of the diacid linker is not possible, and a mixture of nucleoside hemiesters, S.9, and diesters, S.10, always occurs (Fig. 3.2.3). Fortunately, chromatographic separation of the hemiesters and diesters is not necessary, because only the hemiester can couple to the long-chain alkylamine-controlled-pore glass (LCAACPG) support. Thus the preparation of the HQDA hemiesters is not much more difficult than the synthesis of nucleoside-3'- $O$-succinyl hemiesters. Coupling of the HQDA hemiesters to LCAA-CPG is performed in the same manner as the succinyl compounds.

\section{Materials}

5 '-Dimethoxytrityl-N-protected deoxyribonucleoside (see recipe)

Hydroquinone- $O, O^{\prime}$-diacetic acid (HQDA; Lancaster Synthesis)

4-Dimethylaminopyridine (DMAP), reagent grade

1-(3-Dimethylaminopropyl)-3-ethylcarbodiimide hydrochloride (DEC; see recipe)

Triethylamine, reagent grade

Anhydrous pyridine, for coupling reactions (see recipe)

Toluene, reagent grade

Chloroform, reagent grade

$5 \%(\mathrm{v} / \mathrm{v})$ methanol/chloroform, for TLC analysis

Saturated aqueous sodium bicarbonate solution

Anhydrous magnesium sulfate

Pyridine, reagent-grade, for washing supports

AG 50W-X4 $\mathrm{H}^{+}$form cation-exchange resin (Bio-Rad)

LCAA-CPG beads (other amino-derivatized nonswelling support materials may also be used; see recipe)

Methanol, reagent grade

Dichloromethane, reagent grade

Cap A capping solution (see recipe)

Cap B capping solution (see recipe) 
50- and $100-\mathrm{mL}$ round-bottom flasks

Magnetic stir bar

Pasteur pipet

Fluorescent silica gel thin-layer chromatography (TLC) plates, $\sim 2.5 \mathrm{~cm} \times 10 \mathrm{~cm}$ (Merck 60 or similar)

Covered jar for TLC

Shortwave UV (254-nm) lamp

Separatory funnel

Shaker, wrist action or similar

Rotary evaporator

Additional reagents and solutions for dimethoxyl derivatization to assay for amino and hydroxyl groups present (see Support Protocol 2; optional) and trityl analysis (see Support Protocol 1)

CAUTION: Carbodiimide reagents, such as DEC, can cause severe skin or eye irritation and allergic reactions. Use appropriate skin and eye protection, and wipe up all work areas and utensils with a wet cloth immediately after use (DEC is water soluble).

\section{Synthesize nucleoside-3'-O-HQDA hemiesters}

1. Combine in a $100-\mathrm{mL}$ round-bottom flask with a magnetic stir bar:

10 mmol 5'-dimethoxytrityl-N-protected deoxyribonucleoside $3.39 \mathrm{~g}(15 \mathrm{mmol}) \mathrm{HQDA}$

$122 \mathrm{mg}(1 \mathrm{mmol})$ DMAP

$2.88 \mathrm{~g}(15 \mathrm{mmol})$ DEC.

Add $0.8 \mathrm{~mL}$ triethylamine and $50 \mathrm{~mL}$ anhydrous pyridine. Seal the flask and stir overnight at room temperature.

2. Remove 50 to $100 \mu \mathrm{L}$ of the solution using a Pasteur pipet and place in a $50-\mathrm{mL}$ round-bottom flask. Add $\sim 1$ to $2 \mathrm{~mL}$ toluene and evaporate to dryness using a rotary evaporator.

3. Redissolve in $\sim 100$ to $200 \mu \mathrm{L}$ chloroform.

4. Spot the sample near the bottom edge of an $\sim 2.5 \times 10-\mathrm{cm}$ TLC plate, alongside samples of the starting nucleoside and HQDA. Place the TLC plate in a jar containing a small amount of $5 \%(\mathrm{v} / \mathrm{v})$ methanol/chloroform (the liquid should be $\sim 2$ to $4 \mathrm{~mm}$ deep), and allow the solvent to rise most of the way up the TLC plate.

5. Remove plate and examine under a shortwave UV (254-nm) lamp.

The desired hemiester will appear as a UV-absorbing spot near the baseline, and the diester product will be a UV-absorbing spot near the top of the plate. The starting nucleoside will migrate to near the middle of the plate. The coupling reaction is complete when all the starting nucleoside is converted into the faster-(diester) and slower-migrating (hemiester) products.

If more than a trace of starting nucleoside is visible, add more DEC (2 to $5 \mathrm{mmol}$ ) to the reaction, and continue stirring for another day. Repeat steps 2 to 5.

6. When TLC shows complete disappearance of the starting nucleoside, concentrate the solution on the rotary evaporator until a thick oil forms.

7. Redissolve the oil in $\sim 200 \mathrm{~mL}$ chloroform and transfer to a separatory funnel. Wash the solution twice with $\sim 100 \mathrm{~mL}$ saturated aqueous sodium bicarbonate.

CAUTION: Carbon dioxide gas is released during this extraction step. Shake the separatory funnel slowly with frequent venting until gas evolution stops. 
8. Wash the chloroform solution three times with $\sim 100 \mathrm{~mL}$ water each time. Collect the chloroform phase and discard the aqueous phase.

Do not shake the separatory funnel vigorously at this step, because the mixture will form an emulsion that is slow to separate. Invert the funnel slowly to mix the phases. If an inseparable emulsion does form, then either (for small volumes) centrifugation or (for large volumes) precipitation by addition of hexanes followed by filtration and redissolution of the sticky precipitate back into chloroform can be performed.

9. Add the chloroform solution to $\sim 5 \mathrm{~g}$ anhydrous magnesium sulfate, and mix to remove residual moisture from the solution. Filter off the magnesium sulfate, wash with a small amount of chloroform, and then evaporate the chloroform solution to dryness using the rotary evaporator. A light brown foam should form and solidify.

The crude product, which contains a mixture of diester and nucleoside hemiester sodium salt, is suitable for coupling directly to LCAA-CPG using the DEC/pyridine coupling procedure that follows. The sodium salt, however, may not be sufficiently soluble in other solvents for other types of coupling reactions. In this case, the sodium salt can be easily converted into the more soluble pyridinium salt by the procedure outlined in step 10.

10. Conversion into pyridinium salt (optional): Dissolve the crude sodium salt in $\sim 50$ to $100 \mathrm{~mL}$ of reagent-grade pyridine, and add 2 eq AG 50W-X4 $\mathrm{H}^{+}$cation-exchange resin. Stir $\sim 5$ to $10 \mathrm{~min}$; then filter off the ion-exchange resin. Evaporate the pyridine solution to dryness; a light brown foam will form and solidify. Dry under vacuum overnight to remove excess pyridine.

Store in sealed, moisture-proof containers at $-20^{\circ} \mathrm{C}$. Samples are stable for at least 1 to 2 years. Older samples should be checked by TLC to determine the extent of decomposition, if any.

\section{Couple nucleoside 3'-O-HQDA hemiesters to LCAA-CPG}

11. If the amino-group loading of the starting support is not known: Verify the support's capacity by dimethoxyl derivatization to determine the number of amino and hydroxyl groups present (see Support Protocol 2) before starting the nucleoside derivatization.

12. Combine in a round-bottom flask:

$5.0 \mathrm{~g} \mathrm{LCAA}-\mathrm{CPG}$

$2 \mathrm{~g}$ (2.0 mmol) crude nucleoside $3^{\prime}$ - $O$-HQDA hemiester (step 9 or 10 )

$122 \mathrm{mg}(0.1 \mathrm{mmol})$ DMAP

$1.44 \mathrm{~g}(7.5 \mathrm{mmol})$ DEC.

Add $1 \mathrm{~mL}$ triethylamine and $30 \mathrm{~mL}$ anhydrous pyridine and quickly seal the flask. Shake overnight at room temperature.

13. In the fume hood, filter off the support and wash successively with $\sim 25$ to $50 \mathrm{~mL}$ reagent-grade pyridine, $\sim 50$ to $100 \mathrm{~mL}$ methanol, and finally $\sim 100$ to $150 \mathrm{~mL}$ dichloromethane. Allow to dry at room temperature.

14. Remove a small aliquot for trityl analysis to determine the nucleoside loading (see Support Protocol 1).

15. If the nucleoside loading is acceptable, return the support to a clean flask, and add equal amounts $(\sim 25 \mathrm{~mL})$ of Cap A and Cap B capping solutions. Seal the flask and shake $1 \mathrm{hr}$ at room temperature.

Attachment of Nucleosides to Solid-Phase Supports

16. Filter off the support and wash with $\sim 50$ to $100 \mathrm{~mL}$ methanol and then $\sim 100$ to 150 $\mathrm{mL}$ dichloromethane. Allow to dry at room temperature. 
17. Verify the nucleoside loading by performing trityl analysis (see Support Protocol 1) on a sample of the final product.

Supports derivatized with the Q-linker have been stored for 3 years at room temperature without any significant loss of nucleoside.

\section{NUCLEOSIDE COUPLING TO HYDROQUINONE- $O, O^{\prime}$-DIACETIC ACID DERIVATIZED LCAA-CPG}

In this protocol HQDA, S.8, is coupled to LCAA-CPG, S.1, to give a carboxyl-derivatized support, S.12 (Fig. 3.2.4). This coupling reaction must be performed in the presence of a coupling reagent because HQDA is not available as an acid anhydride (as succinic anhydride is for use in succinylation approaches). In addition, the coupling reactions involving immobilized HQDA-especially the formation of esters to the secondary hydroxyl group of protected nucleosides, S.6-are best performed using a more reactive coupling reagent than the carbodiimide used in the above protocols. Therefore, $2-(1 \mathrm{H}-$ benzotriazol-1-yl)-1,1,3,3-tetramethyluronium hexafluorophosphate (HBTU), which is a coupling reagent developed for peptide synthesis (Dourtoglou et al., 1984), is used instead of DEC along with an increased amount of DMAP. Another advantage to using HBTU is that anhydrous acetonitrile can be substituted for pyridine as the solvent.

Additional Materials (also see Basic Protocol 2)

2-(1H-Benzotriazol-1-yl)-1,1,3,3-tetramethyluronium hexafluorophosphate (HBTU)

Anhydrous diisopropylethylamine (DIEA; see recipe)

Anhydrous acetonitrile (see recipe)

Acetonitrile, reagent grade

Screw-capped glass vials or round-bottom flasks

Syringes

Buchner funnel

Whatman no. 1 filter paper

Additional reagents and solutions for $N$-monomethoxytrityl-6-amino-1-hexanol assay of carboxylic acid loading (see Support Protocol 4) and trityl analysis (see Support Protocol 1)

CAUTION: All coupling reagents, such as HBTU, can potentially cause adverse reactions and lead to severe allergic reactions. Although specific data on HBTU are not available, safe laboratory practices (i.e., no skin or eye contact or inhalation of powders) should always be followed when handling this type of reagent.

\section{Couple HQDA to LCAA-CPG}

1. Combine in a glass screw-capped vial or small glass-round bottom flask:

$$
\begin{aligned}
& 1.0 \mathrm{~g} \mathrm{LCAA}-\mathrm{CPG} \\
& 90 \mathrm{mg}(0.4 \mathrm{mmol}) \mathrm{HQDA} \\
& 46 \mathrm{mg}(0.38 \mathrm{mmol}) \text { DMAP } \\
& 144 \mathrm{mg}(0.36 \mathrm{mmol}) \mathrm{HBTU} .
\end{aligned}
$$

Via syringe, add $140 \mu \mathrm{L}(0.8 \mathrm{mmol})$ DIEA and $10 \mathrm{~mL}$ anhydrous acetonitrile. Seal the vial and shake $2 \mathrm{hr}$ at room temperature.

2. Filter off the support using a Buchner funnel and wash the LCAA-CPG successively with $\sim 10$ to $20 \mathrm{~mL}$ acetonitrile, $\sim 10$ to $20 \mathrm{~mL}$ methanol, and finally $\sim 20$ to $30 \mathrm{~mL}$ dichloromethane. Allow the support to dry at room temperature. 
3. Verify the extent of the HQDA derivatization by performing the $N$-monomethoxytrityl-6-amino-1-hexanol assay of carboxylic acid loading (see Support Protocol 4).

A carboxyl loading of 30 to $40 \mu \mathrm{mol} / \mathrm{g}$ is typical. Supports can be stored indefinitely at room temperature.

Store the support at room temperature in a sealed glass vial.

\section{Couple nucleoside to the HQDA-derivatized LCAA-CPG}

4. Combine in a screw-capped glass vial or small glass round-bottom flask:

$1.0 \mathrm{~g}$ HQDA-derivatized LCAA-CPG

$0.2 \mathrm{~g}(0.2 \mathrm{mmol}) 5^{\prime}$-dimethoxytrityl-N-protected deoxyribonucleoside

$24 \mathrm{mg}(0.2 \mathrm{mmol})$ DMAP

$76 \mathrm{mg}(0.2 \mathrm{mmol})$ HBTU.

Via syringe, add $140 \mu \mathrm{L}(0.8 \mathrm{mmol})$ DIEA and $5 \mathrm{~mL}$ anhydrous acetonitrile. Quickly seal the vial, and shake 2 to $3 \mathrm{hr}$ at room temperature.

5. Filter off the support using a Buchner funnel and wash successively with $\sim 10$ to 20 $\mathrm{mL}$ acetonitrile, $\sim 10$ to $20 \mathrm{~mL}$ methanol, and finally $\sim 10$ to $20 \mathrm{~mL}$ dichloromethane. Allow the support to dry at room temperature.

6. Determine the nucleoside loading by trityl analysis of a small sample of support (see Support Protocol 1).

\section{Cap unreacted amino groups}

7. Return the support into a clean vial or flask and add equal amounts $(\sim 5 \mathrm{~mL})$ of Cap A and Cap B capping reagents. Seal the vial and shake $1 \mathrm{hr}$ at room temperature.

8. Filter off the support and wash with $\sim 10$ to $20 \mathrm{~mL}$ methanol and then with $\sim 10$ to 20 $\mathrm{mL}$ dichloromethane. Allow to dry at room temperature.

9. Determine the nucleoside loading by trityl analysis of a small portion of the final product (see Support Protocol 1).

Label the support with this loading value and the date of synthesis, and store at room temperature in a sealed screw-capped vial. Supports can be kept for several years.

SUPPORT PROTOCOL 1

Attachment of Nucleosides to Solid-Phase Supports

\section{QUANTITATIVE TRITYL ANALYSIS OF SURFACE LOADING OF DERIVATIZED SUPPORTS}

This procedure describes how the surface loading of supports containing trityl groups can be determined by colorimetric analysis. This method detects the $5^{\prime}$-trityl protecting group on nucleoside-derivatized supports or trityl groups added to derivatize aminoor hydroxyl-functionalized supports (see Support Protocol 2). This assay is based on the fact that acid-labile dimethoxytrityl (from nucleoside-derivatized supports) or monomethoxytrityl protecting groups (from $N$-monomethoxytrityl-6-amino-1-hexanol-derivatized supports) can be rapidly and quantitatively hydrolyzed to form intensely orangeand yellow-colored solutions.

\section{Materials}

Derivatized LCAA-CPG support (see Basic Protocols 1 and 2 and Alternate Protocols 1 and 2)

Detritylation reagent (see recipe) 
$10-\mathrm{mL}$ volumetric flask

Analytical balance accurate to $0.1 \mathrm{mg}$

Quartz cuvettes

UV/visible spectrophotometer or colorimeter

1. Tare an empty $10-\mathrm{mL}$ volumetric flask on an analytical balance.

2. Place $\sim 4 \mathrm{mg}$ dry, derivatized LCAA-CPG support directly into the flask and weigh. Record the weight to at least 2 significant figures.

3. Add detritylation reagent to the volumetric flask to the mark. Seal the flask and mix. An orange-colored dimethoxytrityl cation (yellow if monomethoxytrityl groups are being measured) will immediately form.

The orange color is stable if moisture contamination and evaporation are avoided and the solution can be kept at room temperature indefinitely.

4. Fill a reference cuvette with detritylating reagent or, if using a single-beam spectrophotometer, run a baseline.

The dichloromethane solvent requires quartz rather than disposable polystyrene cuvettes.

5. Measure the spectrum of the sample from 400 to $600 \mathrm{~nm}$. Record the absorbance of the peak maximum at $\sim 503$ to $505 \mathrm{~nm}$ (dimethoxytrityl group) or $\sim 470 \mathrm{~nm}$ (monomethoxytrityl group).

If the absorbance exceeds the maximum reliable value for the spectrophotometer (usually 2 to 3 absorbance units), prepare another sample with less support or perform a serial dilution using detritylation reagent as the diluent.

6. Calculate the loading of the support using the following equation (for a cuvette with a 1-cm path length):

$$
\text { Loading }=\frac{\text { volume } \times \text { absorbance }}{\varepsilon} \times \frac{1000}{\text { weight }}
$$

where loading is in micromoles per gram, the solution volume in milliliters, and the support weight is in milligrams; and $\varepsilon$ is the extinction coefficient of either the dimethoxytrityl group $\left(76 \mathrm{~mL} \mathrm{~cm}^{-1} \mu \mathrm{mol}^{-1}\right)$ or the monomethoxytrityl group $(56 \mathrm{~mL}$ $\left.\mathrm{cm}^{-1} \mu \mathrm{mol}^{-1}\right)$.

\section{DIMETHOXYTRITYL DERIVATIZATION OF AMINO- AND/OR HYDROXYL-FUNCTIONALIZED SUPPORTS}

A variety of methods have been developed to determine the primary amino group content of insoluble supports (Horn and Novak, 1987). The most convenient method, however, uses the dimethoxytrityl chloride/tetrabutylammonium perchlorate reagent developed for the solid-phase tritylation of nucleosides and nucleotides (Reddy et al., 1987). This reagent will also tritylate amino groups, and it has been used to monitor coupling efficiency in solid-phase peptide synthesis (Reddy and Voelker, 1988). This is a very fast, sensitive, and general method for determining the number of amino and hydroxyl groups on insoluble supports.

\section{Materials}

LCAA-CPG support

$0.25 \mathrm{M}$ dimethoxytrityl (see recipe) chloride stock solution (see recipe)

$0.25 \mathrm{M}$ tetrabutylammonium perchlorate stock solution (see recipe)

Methanol, reagent grade 
SUPPORT PROTOCOL 3

Attachment of Nucleosides to Solid-Phase Supports
Dichloromethane, reagent grade

Screw-capped glass vials

1. Place $\sim 10$ to $20 \mathrm{mg}$ of LCAA-CPG support in a small screw-capped glass vial and add equal amounts $(\sim 500 \mu \mathrm{L})$ of $0.25 \mathrm{M}$ dimethoxytrityl chloride and $0.25 \mathrm{M}$ tetrabutylammonium perchlorate. Seal the vial and shake $10 \mathrm{~min}$ at room temperature.

2. Filter off the support and wash first with 10 to $20 \mathrm{~mL}$ dichloromethane, then with 10 to $20 \mathrm{~mL}$ methanol, and finally with 20 to $30 \mathrm{~mL}$ dichloromethane again.

The methanol wash is important to remove any adsorbed trityl reagent from the support.

3. Allow the support to dry and then perform the colorimetric trityl analysis (see Support Protocol 1).

\section{DETERMINATION OF CARBOXYLIC ACID GROUPS ON INSOLUBLE SUPPORTS BY $p$-NITROPHENOL DERIVATIZATION}

The amount of carboxylic acid attached to the surface on an insoluble support can be determined by titration, but this consumes large amounts (gram quantities) of support. A better and more sensitive method is to derivatize the support with an appropriate reporter molecule. $p$-Nitrophenol is a convenient and readily available label that has been widely used. When this label is removed from the support, by hydrolysis with aqueous sodium hydroxide, a yellow-green solution is produced that can be quantitated by colorimetry (Matteucci and Caruthers, 1981; Damha et al., 1990). Although the extinction coefficient for the $p$-nitrophenoxide group is only one-fifth as large as for the dimethoxytrityl cation (see Support Protocol 1), this test is useful for measuring the succinic acid surface loading produced in Alternate Protocol 1. This method does not, however, work with HQDA-derivatized LCAA-CPG (see Support Protocol 4 instead).

\section{Additional Materials (see Basic Protocol 1 and Alternate Protocol 1)}

$p$-Nitrophenol, reagent grade

$0.1 \mathrm{M}$ aqueous $\mathrm{NaOH}$ solution

$\mathrm{N}$-Monomethoxytrityl-6-amino-1-hexanol

$10-\mathrm{mL}$ volumetric flask

Analytical balance

UV/visible spectrophotometer or colorimeter

Additional reagents and solutions for DEC/DMAP coupling (see Alternate

Protocol 1) and trityl analysis (see Support Protocol 1)

1. Combine in a small screw-capped glass vial or round-bottom flask:

$1.0 \mathrm{~g}$ succinylated LCAA-CPG

$14 \mathrm{mg}(0.1 \mathrm{mmol}) p$-nitrophenol

$190 \mathrm{mg}(0.1 \mathrm{mmol})$ DEC.

Via syringe, add $40 \mu \mathrm{L}$ triethylamine and $1 \mathrm{~mL}$ anhydrous pyridine. Seal the vial, and shake overnight at room temperature.

2. Filter off the support and wash sequentially with $5 \mathrm{~mL}$ pyridine, $10 \mathrm{~mL}$ methanol, and 10 to $20 \mathrm{~mL}$ dichloromethane. Leave the support uncovered at room temperature to dry.

The capping steps (see Alternate Protocol 1, steps 10 to 15) are unnecessary or this protocol. 
3. Accurately weigh $\sim 10 \mathrm{mg}$ of support directly into a 10 -mL volumetric flask. Add 0.1 $\mathrm{M} \mathrm{NaOH}$ solution to the mark and mix.

4. Determine the absorbance of the solution at $400 \mathrm{~nm}$. Calculate the support loading using an extinction coefficient $(\varepsilon)$ of $15.7 \mathrm{~mL} \mathrm{~cm}^{-1} \mu \mathrm{mol}^{-1}$ for the nitrophenoxide group; see Support Protocol 1, step 6, for formula.

\section{DETERMINATION OF CARBOXYLIC ACID GROUPS ON INSOLUBLE SUPPORTS BY MONOMETHOXYTRITYL DERIVATIZATION}

This protocol is an alternate to Support Protocol 3. Unlike Support Protocol 3, this method can be used to determine the carboxyl loading of both succinyl- and HQDA-derivatized LCAA-CPG. This method requires synthesis of a custom reporter molecule, $\mathrm{N}$ monomethoxytrityl-6-amino-1-hexanol, developed by Pon and Yu (1997a). The carboxyl loading determination is more sensitive, because the yellow color produced from the monomethoxytrityl group (in acid solution; i.e., see Support Protocol 1) is more than three times as intense as that produced by the $p$-nitrophenoxide group.

Additional Materials (see Basic Protocol 2 and Alternate Protocol 2)

6-Amino-1-hexanol

Monomethoxytrityl chloride

$\mathrm{N}$-Monomethoxytrityl-6-amino-1-hexanol (see recipe)

Silica gel, Merck 60, for flash chromatography

Glass chromatography column for flash chromatography, $\sim 4$ to $5 \mathrm{~cm}$ diameter $\times$ $\sim 50$ to $60 \mathrm{~cm}$ long

Silica-gel TLC plates (Merck, $\sim 2.5 \times 8 \mathrm{~cm}$ )

CAUTION: All coupling reagents, such as HBTU, can potentially cause adverse reactions and lead to severe allergic reactions. Although specific data on HBTU are not available, safe laboratory practices (i.e., no skin or eye contact or inhalation of powders) should always be followed when handling this type of reagent.

1. Dissolve $2.42 \mathrm{~g}(20 \mathrm{mmol})$ 6-amino-1-hexanol and $6.37 \mathrm{~g}(20 \mathrm{mmol})$ monomethoxytrityl chloride in $100 \mathrm{~mL}$ anhydrous pyridine, and stir 1 to 2 days at room temperature.

2. Concentrate the solution on a rotary evaporator to 10 to $15 \mathrm{~mL}$.

3. Redissolve in $75 \mathrm{~mL}$ chloroform, wash with $25 \mathrm{~mL}$ aqueous sodium bicarbonate solution, and wash twice with $25 \mathrm{~mL}$ water. Dry over anhydrous magnesium sulfate.

4. Concentrate the chloroform solution to an oil.

5. Apply to a flash chromatography column packed $\sim 20 \mathrm{~cm}$ high with Merck 60 silica gel. Begin the purification by eluting the column with $5 \%$ methanol/chloroform and collect the eluate in $\sim 50$ - to 100 -mL fractions.

6. Monitor the separation by spotting a small amount ( 2 to $5 \mu \mathrm{L})$ of each fraction on a silica-gel TLC plate $(\sim 2.5 \times 8 \mathrm{~cm})$. Develop the plate in $20 \%$ methanol/chloroform and detect the products either by UV or by spraying with detritylation reagent and then gently warming the plate (e.g., with a hair dryer) for a few seconds: monomethoxytrityl-containing products will appear as yellow spots. Once all the unreacted monomethoxytrityl chloride has eluted from the column or when the desired product begins to appear, change the solvent to $15 \%$ methanol/chloroform. 
Initially, hydrolyzed, unreacted monomethoxytrityl chloride will appear as an impurity moving near the solvent front. The desired product will have a much slower mobility $\left(R_{f} \sim 0.15\right)$. Unreacted aminohexanol will remain bound to the silica gel.

7. Combine fractions containing the desired product. Evaporate on a rotary evaporator to obtain pure $N$-monomethoxytrityl-6-amino-1-hexanol as a thick brown liquid (5.5 g, $71 \%$ yield).

8. Combine in a screw-capped glass vial or small glass round-bottom flask:

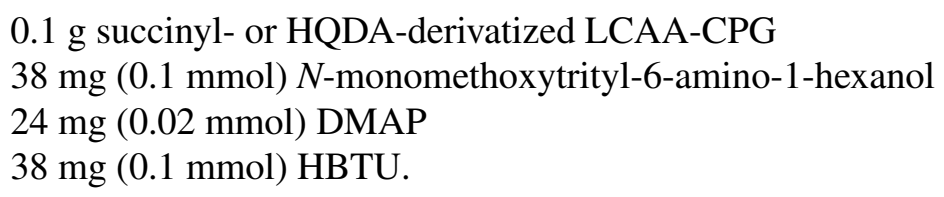

Via syringe, add $14 \mu \mathrm{L}$ DIEA $(0.08 \mathrm{mmol})$ and $\sim 0.5 \mathrm{~mL}$ anhydrous acetonitrile. Quickly seal the vial and shake 2 to $3 \mathrm{hr}$ at room temperature.

9. Filter off the support using a Buchner funnel, and wash successively with $\sim 10$ to 20 $\mathrm{mL}$ acetonitrile, $\sim 10$ to $20 \mathrm{~mL}$ methanol, and finally $\sim 20$ to $30 \mathrm{~mL}$ dichloromethane. Allow the support to dry uncovered at room temperature.

10. Determine the monomethoxytrityl loading by trityl analysis (see Support Protocol 1).

NOTE: The monomethoxytrityl group generates a yellow rather than an orange color, and the measurement must be made with the wavelength and extinction coefficient specified in Support Protocol 1.

\section{REAGENTS AND SOLUTIONS}

Use deionized, distilled water in all recipes and protocol steps. For common stock solutions, see APPENDIX 2A; for suppliers, see SUPPLIERS APPENDIX.

\section{Anhydrous acetonitrile}

Distill from calcium hydride or obtain from commercially available "low-water" reagents suitable for automated oligonucleotide synthesis. Store over molecular sieves in tightly sealed bottles. Discard or redistill after each day's use.

\section{Anhydrous diisopropylethylamine (DIEA)}

Purchase as the $99.5 \%$ redistilled grade (Aldrich), and keep anhydrous by storage over type 4A molecular sieves. Store in septum-sealed bottles at room temperature, protected from light.

\section{Anhydrous pyridine}

Prepare by distillation from calcium hydride, and keep anhydrous by storage over type $4 \mathrm{~A}$ molecular sieves in tightly sealed bottles. Store up to 1 year at room temperature.

Alternatively, this may be purchased ready to use in Sure/Seal bottles (Aldrich).

\section{Cap $A$ and Cap B capping reagents}

Cap A: $94 \mathrm{~mL} 1 \mathrm{M}$ acetic anhydride/116 mL 1 M 2,6-lutidine or pyridine in $790 \mathrm{~mL}$ tetrahydrofuran (THF). Store indefinitely at room temperature.

Attachment of Nucleosides to Solid-Phase Supports
Cap B: $160 \mathrm{~mL} 2 \mathrm{M} \mathrm{N}$-methylimidazole in $840 \mathrm{~mL}$ THF. Store indefinitely at room temperature.

These are the same reagents used as capping reagents on automated DNA synthesizers, and may be purchased commercially. 
$5 \%$ Dichloroacetic acid (v/v) or $3 \%$ trichloroacetic acid (w/v) in 1,2-dichloroethane or dichloromethane. Store indefinitely at room temperature.

There is no significant difference between these reagents in the trityl analysis, although dichloroacetic acid reagents are more suitable for the synthesis of long oligonucleotides. To reduce solvent vapors, it is recommended that the less volatile 1,2-dichloroethane be substituted for the more commonly used dichloromethane. Detritylation reagent may be purchased commercially (sometimes referred to as "deblock" reagent).

CAUTION: Many older references suggest the use of perchloric acid solutions to dilute the trityl colors. Such a strong acid is not required, and for safety reasons, perchloric acid solutions should not be used. The same detritylation reagent used on the DNA synthesizers can be used to dilute the colors for measurement.

\section{Dimethoxytrityl chloride stock solution, $0.25 \mathrm{M}$}

Dissolve $847 \mathrm{mg}(2.5 \mathrm{mmol})$ dimethoxytrityl chloride in $10 \mathrm{~mL}$ dichloromethane. Store in a sealed glass vial up to 3 to 4 weeks at room temperature.

\section{5'-Dimethoxytrityl-N-protected nucleosides}

Purchase 5'-dimethoxytrityl nucleosides with a free 3'-OH or, for ribonucleosides, free $2^{\prime}$ - and $3^{\prime}-\mathrm{OH}$ groups (Chem-Impex, Sigma, and ChemGenes). $N^{6}$-benzoyl-5'dimethoxytrityl-2' -deoxyadenosine, $N^{4}$-benzoyl- $5^{\prime}$-dimethoxytrityl- $2^{\prime}$-deoxycytidine, $N^{2}$-isobutyryl-5'-dimethoxytrityl-2'-deoxyguanosine, and $5^{\prime}$-dimethoxytritylthymidine are the most commonly used standard nucleosides and are compatible with the protocols described in this unit. Store indefinitely at room temperature.

\section{5'-Dimethoxytrityl-N-protected nucleoside succinyl hemiesters}

Purchase nucleosides with the standard protecting groups $-N^{6}$-benzoyl- $5^{\prime}$-dimethoxytrityl-2'-deoxyadenosine- $3^{\prime}$ - $O$-succinate, $N^{4}$-benzoyl- $5^{\prime}$-dimethoxytrityl$2^{\prime}$-deoxycytidine-3'-O-succinate, $N^{2}$-isobutyryl-5'-dimethoxytrityl-2'-deoxy-guanosine$3^{\prime}$ - $O$-succinate, and $5^{\prime}$-dimethoxytritylthymidine- $3^{\prime}$ - $O$-succinate (Chem-Impex and Sigma). Store indefinitely at room temperature.

Only the standard succinates are available for purchase; more unusual or complicated succinates must be made up.

\section{1-(3-Dimethylaminopropyl)-3-ethylcarbodiimide hydrochloride (DEC)}

Also referred to as 3-ethyl-1-(3-dimethylaminopropyl)-carbodiimide (EDC) and water soluble carbodiimide (WSC). Readily available from commercial suppliers. Store indefinitely at $-20^{\circ} \mathrm{C}$.

\section{Long-chain alkylamine-controlled-pore glass (LCAA-CPG)}

Available in a variety of pore and particle sizes and sieved into three sets of particle sizes: 80 to 120 mesh ( 125 to $177 \mu \mathrm{m}), 120$ to 200 mesh ( 74 to $125 \mu \mathrm{m}$ ), and 200 to 400 mesh ( 37 to $74 \mu \mathrm{m}$ ); the two largest size ranges are the most suitable for oligonucleotide synthesis. Generally, 500- $\AA$-diameter pores are used for oligonucleotides of up to $\sim 50$ bases in length, and 1000 - $\AA$-diameter pores are required for longer oligonucleotides. Store indefinitely at room temperature.

\section{N-Monomethoxytrityl-6-amino-1-hexanol}

Prepare from monomethoxytrityl chloride and 6-amino-1-hexanol (see Support Protocol 4 or Pon and Yu, 1997a). Store indefinitely at room temperature.

\section{Tetrabutylammonium perchlorate stock solution, $0.25 \mathrm{M}$}

Dissolve $854 \mathrm{mg}$ ( $2.5 \mathrm{mmol}$ ) tetrabutylammonium perchlorate (Sigma) in $0.3 \mathrm{~mL}$ 2,6-lutidine and $9.7 \mathrm{~mL}$ dichloromethane. Store in a sealed glass vial up to 3 to 4 weeks at room temperature. 


\section{COMMENTARY}

\section{Background Information}

The succinic acid linker was one of the earliest linkages used to immobilize nucleosides to insoluble supports (Ogilvie and Kroeker, 1971; Yip and Tsou, 1971), and it has remained widely used since. Although a variety of different linkages have been developed for specialized applications (UNIT 3.1; Pon, 1993), routine oligonucleotide synthesis has been well served by the succinyl linker, primarily because succinic anhydride, S.4, does not need any activation to react with hydroxyl (or amino) groups and selectively produces only hemiesters (or amides). The low cost and easy handling of this reagent are also advantageous.

Once the anhydride has been opened, however, coupling to the terminal free carboxylic acid group is more difficult. This group requires activation by conversion to an acid chloride (Ogilvie and Kroeker, 1971; Sharma et al., 1992), an activated ester, or a symmetrical anhydride (Gait et al., 1980) or by reaction with a coupling reagent such as dicyclohexylcarbodiimide (DCC; Chow et al., 1981; Matteucci and Caruthers, 1981; Montserrat et al., 1993). Because acid chlorides are difficult to work with and the DCC coupling reagent does not perform well with LCAA-CPG supports, most early work was performed using activated nucleoside-3'-O-succinyl pentachlorophenyl (Miyoshi et al., 1980), $p$-nitrophenyl (Koster et al., 1983), or pentafluorophenyl esters (Efimov et al., 1993). These intermediates, however, require an additional coupling step to prepare, and the support derivatization reactions are slow. Later it was discovered that the carbodiimide reagent DEC could rapidly couple unactivated nucleoside succinates directly to LCAA-CPG (Pon et al., 1988). Given that coupling reactions with DEC are completed in as little as $1 \mathrm{hr}$, this technique has become the preferred approach.

Recently, however, improved coupling methods have been described using diisopropylcarbodiimide and hydroxybenzotriazole (Bhongle and Tang, 1995) or DCC and 3,4-dihydro-3-hydroxy-4-oxo-1,2,3-benzotriazine (Walsh et al., 1997), which allow satisfactory derivatization of LCAA-CPG supports. Other new coupling reagents have also been used to speed up the derivatization reactions. Use of an oxidation-reduction coupling reagent combination, triphenylphosphine and 2,2'dithiobis(5-nitropyridine), reduces the coupling time to between 2 and 30 min (Kumar et al., 1996). Extremely fast derivatization (4 sec) is obtained by using either uronium- or phosphonium-coupling reagents and DMAP (Pon and Yu, 1997b); however, none of these methods has been widely adopted.

Another benefit of the DEC coupling reagent is that it can also be used to create a secondary ester linkage between the $3^{\prime}$ (or $2^{\prime}$ ) hydroxyl group of a nucleoside and a succinylated support (Damha et al., 1990). Because forming a secondary ester is more difficult than creating a primary amide, the coupling reaction is slower and support loadings are often not as high as obtained from coupling a pre-formed nucleoside-3'-succinate to LCAA-CPG. Not having to prepare nucleoside succinates is a significant advantage, however, especially when limited starting materials are available.

Recently, the huge success of solid-phase oligonucleotide synthesis has created a great demand for large numbers of synthetic oligonucleotides, and a number of productivity enhancements have been implemented. New high-throughput DNA synthesizers have been developed, and more labile protecting groups and faster deprotection conditions have been introduced to speed up processing times. A significant increase in productivity can also be obtained by replacing the succinyl linker with a more rapidly cleavable linker. This is particularly so with instrumentation that performs the cleavage step automatically after completion of oligonucleotide synthesis. In this case, the automated synthesizer essentially remains idle for the 60 to $90 \mathrm{~min}$ required for the cleavage of the succinyl linker arm by ammonium hydroxide. A very labile oxalic acid linker arm, which can be cleaved in only a few seconds, was introduced for the synthesis of base-sensitive oligonucleotide modifications (Alul et al., 1991). Unfortunately, the oxalyl linker proved to be too labile for routine use, because of spontaneous cleavage during storage at room temperature. Therefore, a linker arm that was more stable than oxalic acid but more easily cleaved than succinic acid was sought. This resulted in the introduction of hydroquinone$O, O^{\prime}$-diacetic acid (HQDA or Q-linker), $\mathbf{S . 8}$, as a new all-purpose linker arm (Pon and Yu, 1997a). Using Q-linker, ammonium hydroxide cleavage requires only 2 min for oligodeoxyribonucleotides, $5 \mathrm{~min}$ for oligoribonucleotides, and $10 \mathrm{~min}$ for phosphorothioate-modified oligonucleotides. Cleavage can also be performed using a number of other reagents (Table 3.2.1) 


\begin{tabular}{lrrrrr}
\hline & \multicolumn{5}{c}{ Amount of cleavage (\%) at room temperature } \\
\cline { 2 - 6 } \multicolumn{1}{c}{ Reagent } & $1 \mathrm{~min}$ & $5 \mathrm{~min}$ & $15 \mathrm{~min}$ & $60 \mathrm{~min}$ & $t_{1 / 2}$ \\
\hline $40 \%$ aqueous $\mathrm{MeNH} / \mathrm{NH}_{4} \mathrm{OH}, 1: 1(\mathrm{v} / \mathrm{v})$ & 100 & & & & $<10 \mathrm{sec}$ \\
$0.05 \mathrm{M} \mathrm{K}_{2} \mathrm{CO}_{3}$ in $\mathrm{MeOH}$ & 100 & & & & $<10 \mathrm{sec}$ \\
9.3 bar $(140 \mathrm{psi})$ ammonia gas & 94 & 100 & & & $\approx 10 \mathrm{sec}$ \\
Triethylamine trihydrofluoride (neat) & 46 & 100 & & & $\approx 1 \mathrm{~min}$ \\
$1 \mathrm{M}$ tetrabutylammonium fluoride in THF & 36 & 83 & 99 & & $\approx 2 \mathrm{~min}$ \\
$1: 2: 1(\mathrm{v} / \mathrm{v} / \mathrm{v}) t$-butylamine/MeOH/ $\mathrm{H}_{2} \mathrm{O}$ & 97 & & & & $\approx 1 \mathrm{~min}$ \\
$\mathrm{NH}_{3}$ in MeOH $($ saturated) & 27 & 75 & 98 & & $\approx 3 \mathrm{~min}$ \\
$5 \% \mathrm{NH}_{4} \mathrm{OH}$ in $\mathrm{MeOH}$ & 9 & 27 & 63 & 98 & $\approx 11 \mathrm{~min}$ \\
$20 \%$ piperidine/DMF & & & & 15 & $\approx 3 \mathrm{hr}$ \\
$0.5 \mathrm{M}(7.5 \%)$ DBU in pyridine & & & & 7 & $\approx 16 \mathrm{hr}$ \\
$1: 1(\mathrm{v} / \mathrm{v})$ triethylamine/EtOH & & 2 & 4 & 5 & \\
\hline
\end{tabular}

to accommodate base-sensitive oligonucleotides. The stability of HQDA is sufficient for long-term storage at room temperature $(>3$ years) and for virtually complete resistance to oligonucleotide synthesis conditions. Prepacked synthesis columns with Q-linker are now commercially available (Glen Research), and if the current emphasis on faster production and milder deprotection continues, HQDA may eventually replace the succinyl linker.

\section{Compound Characterization}

Characterization of products attached to the surface of insoluble supports is very difficult, if not impossible. This is especially so with a rigid, nonswelling, inorganic support such as controlled-pore glass beads. Therefore, the success of nucleoside derivatization reactions is usually determined indirectly by examining cleavage products. This can range from the comprehensive characterization of nucleoside or oligonucleotide products hydrolyzed from the support to the simple trityl analysis described in this unit. The dimethoxytrityl protecting group, commonly used to block the $5^{\prime}-\mathrm{OH}$ of nucleosides, provides a convenient and highly sensitive marker (Support Protocol 1). There is a 1:1 relationship between the amount of orange-colored dimethoxytrityl cation released and the amount of nucleoside present, so nucleoside loading of any support can be easily determined by a colorimetric measurement. Because the trityl group is easily hydrolyzed (by mild acid) from the support and generates such a distinctive and easily quantitated species, it is much simpler and faster to perform a trityl analysis than a nucleoside analysis.
The trityl results can be quite accurate and reproducible, as long as care is taken to avoid the following three possibilities. First, the trityl colors are most stable in the presence of excess acid. Therefore, dilutions should be performed by adding acidic deprotection reagent and not plain solvent. Second, the orange color is quenched by traces of protic solvents, such as water or alcohols. Thus glassware and cuvettes should be clean and dry before use. Finally, the trityl assay does not distinguish between nucleosides (or trityl groups) that are covalently and noncovalently bound. Thus it is possible for supports that have not been adequately washed to give false trityl results. This is not usually a problem, because most unbound reagents are easily removed by a methanol wash. Positively charged amino supports can, however, sometimes bind negatively charged nucleoside hemiesters strongly enough to resist washing. This is one reason why the derivatization protocols recommend checking the trityl loading both before and after acetylation of the surface amino groups (i.e., the capping step with Cap A and Cap B reagents).

The number of functional groups on the surface of the support can also be determined by trityl analysis, if they can be derivatized with trityl groups. Amino and/or hydroxyl surface loadings are easily determined through the dimethoxytrityl derivatization procedure described in Support Protocol 2 (Reddy and Voelker, 1988). Surface carboxylic acids groups cannot be directly tritylated, so an ester derivative containing a chromophore is made instead. $p$-Nitrophenyl ester derivatives (Matteucci and Caruthers, 1981; Damha et al., 1990) can be used to measure succinic acid groups
Synthesis of Unmodified Oligonucleotides 
(Support Protocol 3), but this method does not work for HQDA groups. Instead, a ligand bearing a monomethoxytrityl group on the amino end of 6-amino-1-hexanol is used as the chromophore (Support Protocol 4). It is generally useful to know the extent of surface modification before proceeding with any nucleoside derivatization. This ensures that the support functionalization was successful and that the desired degree of nucleoside loading can be achieved.

Characterization of nucleoside hemiesters, either succinyl or HQDA, can be most easily performed by thin-layer chromatography (TLC), especially if samples of authentic material are available for comparison. Even if authentic samples are not available, observation of the complete conversion of the starting nucleoside into either slower-moving products (hemiesters) or faster-moving products (diesters) is sufficient for most casual users. TLC analysis of the nucleoside hemiesters along with satisfactory trityl results from the derivatized support are usually sufficient if the identity of the starting nucleosides is well established (i.e., commercially available material). Of course, researchers working with novel compounds will also require the usual NMR, $\mathrm{UV}$, and mass or elemental composition analyses to sufficiently confirm structures for publication.

\section{Critical Parameters and Troubleshooting}

\section{Handling of the support}

Insoluble supports must be handled carefully to prevent particle fracturing and the formation of fines because these small particles can plug filters or create higher back pressures in synthesis columns. Although CPG particles are much more durable than the silica gel supports previously used, it is still important to avoid the use of stirrers (magnetic or otherwise) when performing coupling reactions. A shaker, such as a wrist-action shaker, is the preferred method for keeping reaction mixtures agitated.

\section{Quality reagents and solvents}

Lower-than-expected support loadings are obtained if the coupling reagents (DEC or HBTU) are more than several years old or have been exposed to moisture contamination. These reagents should be kept at $-20^{\circ} \mathrm{C}$ for long-term storage and not opened until warmed to room temperature to prevent contamination from moisture condensation. A more common prob- lem is the presence of moisture in the anhydrous reactions. Both pyridine and acetonitrile are quite hygroscopic, and storage over molecular sieves is strongly recommended. Although the coupling reactions described in this unit are not extremely sensitive to moisture contamination, because a large excess of coupling reagent is used in each protocol, excessive moisture will cause poor results.

\section{Reagent concentration}

One of the most important factors in reactions involving solid-phase supports is the solution concentration, and the easiest way to improve results, without consuming more of the reagents, is to increase the concentration of either nucleoside or coupling reagent by reducing the reaction volume. This method is limited, however, by the volume required to suspend the support. Typically, 4 to $5 \mathrm{~mL}$ of solution per gram of LCAA-CPG is the minimum required. Generally, the protocols described in this unit do not depend strongly on reagent concentration, and reasonable results can be obtained without careful measurement of solvent volume. Researchers should be aware, however, that excessive solvent will lower results through both dilution effects and increased moisture content (if solvents are not completely anhydrous).

\section{Ribonucleosides}

Although, for simplicity, the figures and text in this unit refer only to 2'-deoxyribonucleosides, the coupling procedures are also applicable to ribonucleosides. When a ribonucleoside is attached to an insoluble support, it is of no consequence whether the linkage is through the 2' or 3' hydroxyl group. Therefore, ribonucleosides with a single unprotected $2^{\prime}$ or $3^{\prime}$ hydroxyl group can be used, as well as mixtures of the $2^{\prime}$ and $3^{\prime}$ isomers. It is also possible to use ribonucleosides with both $2^{\prime}$ and $3^{\prime}$ hydroxyl groups unprotected (i.e., only 5'and $\mathrm{N}$-protecting groups). In this case, after attachment of the nucleoside to the support through one hydroxyl group, the adjacent unreacted hydroxyl group is acetylated by the same capping step used to acetylate unreacted amino groups.

\section{HBTU side reaction with amines}

Researchers should also be aware that the HBTU coupling reagent can form a Schiff base with amino groups (Gausepohl et al., 1992; Story and Aldrich, 1994). Therefore, this reagent should never be added to LCAA-CPG in 
the absence of the carboxylic acid component of the coupling reaction (which normally, and very rapidly, reacts preferentially with HBTU). Thus only 0.9 eq of HBTU are used in the protocol for coupling HQDA to LCAA-CPG. Accidental incorporation of $>1$ eq of HBTU into this reaction results in conversion of surface amino groups to unreactive Schiff bases.

\section{Capping of unreacted amino groups on the support}

An essential part of each nucleoside coupling protocol is the blocking or capping of unreacted functional groups on the surface of the support. These unreacted groups are present because the amount of nucleoside added is almost always less than the total capacity of the support. In particular, it is very important to cap off any unreacted amino groups by acetylating them with acetic anhydride. Otherwise they will react with the phosphoramidite reagents delivered during subsequent oligonucleotide synthesis cycles to produce oligonucleotide fragments with a $3^{\prime}$ deletion (i.e., the terminal nucleoside will be missing). These deletion fragments will be attached to the solid support via a $3^{\prime}$-phosphoramidate linkage and can be released from the support by either acidic hydrolysis (cleavage of the phosphoramidate bond) or prolonged ammonium hydroxide hydrolysis (cleavage of the silyl bonds joining the linker arm to the LCAA-CPG surface). Although normal cleavage conditions $\left(\mathrm{NH}_{4} \mathrm{OH}\right.$, $\leq 1 \mathrm{hr}$, room temperature) do not release significant amounts of these $3^{\prime}$-deletion products, they still reduce coupling yields and oligonucleotide quality by steric hindrance and competition for reagents. The effectiveness of the support capping can be determined by comparing the amount of trityl color released from the first nucleoside with the amount of color released after the first phosphoramidite-coupling cycle (Pon et al., 1988). The second amount should be the same as, or slightly less than, the first amount, if coupling occurred only on the immobilized nucleoside (i.e., the yield of the coupling is $\leq 100 \%$ ); however, if the second color is more intense than the first (i.e., apparent coupling yields of $>100 \%$ ), then unwanted coupling to the surface of the support is occurring. In this case, the supports should be capped again before further use.

\section{The pore size of the support}

For rigid, macroporous supports, such as the LCAA-CPG particles described in this unit, the pore size is the most important parameter. This is because the pore size affects the amount of nucleoside loading that can be obtained and the coupling efficiency of subsequent chain extension steps during oligonucleotide synthesis. The smaller the pore size, the greater the surface area and the higher the nucleoside loading. Too small a pore size, however, results in poor coupling efficiency and limits the oligonucleotide length that can be synthesized. LCAACPG supports with 500-A pores are good choices for most oligonucleotide syntheses (shorter than $\sim 50$ bases), and LCAA-CPG supports with 1000-Å pores are recommended for the synthesis of oligonucleotides longer than $\sim 50$ bases (see UNIT 3.1).

\section{Anticipated Results}

The amount of nucleoside attached to the surface of the support is generally not very critical, because most oligonucleotide synthesis columns can produce a great deal more product than required. Generally, however, LCAA-CPG supports are prepared with nucleoside loadings in the 30 - to $40-\mu \mathrm{mol} / \mathrm{g}$ range; and the protocols in this unit should produce supports like these. This amount of loading gives excellent results for all but the very longest oligonucleotides. The greater the nucleoside loading, the more crowded the surface of the support; and this crowding appears to have an effect on coupling efficiency, especially with nucleoside loadings $>50 \mu \mathrm{mol} / \mathrm{g}$. Therefore, it is not necessary, and actually detrimental, to try to obtain nucleoside loadings greater than $\sim 40$ to $50 \mu \mathrm{mol} / \mathrm{g}$.

For very long oligonucleotides (75 to 150 bases), use 1000-Å-pore-sized LCAA-CPG supports with only 5 to $10 \mu \mathrm{mol} / \mathrm{g}$ of nucleoside. The lower nucleoside loading presumably improves coupling efficiency by reducing steric hindrance and increasing the amount of excess coupling reagents. With this low loading, however, the maximum capacity of a synthesis column is reduced. It is also important to ensure that the synthesis reagents are of the highest quality and that the acidic detritylation conditions are minimized (to avoid unwanted depurination and chain cleavage) when preparing long oligonucleotides.

\section{Time Considerations}

The total time required to perform each support derivatization varies between 1 and 4 days; however, the time required for the actual setup and workup is much less. Setup time for most reactions is very short $(<15 \mathrm{~min})$, because the reactants just need to be weighed and added
Synthesis of Unmodified Oligonucleotides

Current Protocols in Nucleic Acid Chemistry 
to a single flask. Workup of the support derivatization reactions is also very fast $(<10 \mathrm{~min})$, because this involves only filtering off the support and washing it with solvent. Use of a vacuum aspirator to pull the solvent through the support during the wash steps makes very quick work. After the final dichloromethane wash recommended for each procedure, the LCAACPG beads are left to dry uncovered at room temperature. Evaporation of the dichloromethane solvent is quite fast, and the beads are usually sufficiently dry for trityl analysis or other use within 10 to $20 \mathrm{~min}$. Also, preparations of the succinyl- or HQDA-derivatized LCAA-CPG supports should be performed in bulk, and the intermediate carboxyl derivatized supports saved for future nucleoside coupling reactions. Thus conversion of the LCAA-CPG support to a carboxylic acid-derivatized support does not need to be repeated each time.

The time required for the synthesis of nucleoside hemiesters (of either succinic acid or HQDA) is more substantial, because these products must be worked up by conventional solution phase methods. An experienced chemist will require at least 2 to 3 days to synthesize and purify each reaction, and this process must be repeated for each nucleoside required. Therefore, these products are best purchased, if synthetic resources are not readily available.

Once the support derivatization methods are mastered, it is very easy to prepare sufficient material for a large number of oligonucleotide syntheses. For example, a 5-g support preparation is sufficient for about $5000.2-\mu \mathrm{mol}-\mathrm{scale}$ syntheses or about 200040 -nmol-scale syntheses.

\section{Literature Cited}

Alul, R.H., Singman, C.N., Zhang, G.R., and Letsinger, R.L. 1991. Oxalyl-CPG-A labile support for synthesis of sensitive oligonucleotide derivatives. Nucl. Acids Res. 19:1527-1532.

Bhongle, N.N. and Tang, J.Y. 1995. A convenient and practical method for derivatization of solid supports for nucleic acid synthesis. Synth. Commun. 25:3671-3679.

Chow, F., Kempe, T., and Palm, G. 1981. Synthesis of oligodeoxyribonucleotides on silica gel support. Nucl. Acids Res. 12:2807-2817.

Damha, M.J., Giannaris, P.A., and Zabarylo, S.V. 1990. An improved procedure for derivatization of controlled pore glass beads for solid-phase oligonucleotide synthesis. Nucl. Acids Res. 18:3813-3821.
Dourtoglou, V., Gross, B., Lambropoulou, V., and Zioudrou, C. 1984. O-Benzotriazolyl$N, N, N^{\prime}, N^{\prime}$-tetramethyluronium hexafluorophosphate as a coupling reagent for the synthesis of peptides of biological interest. Synthesis 572-574.

Efimov, V.A., Kalinkina, A.L., and Chakhmakhcheva, O.G. 1993. Dipentafluorophenyl carbonate-A reagent for the synthesis of oligonucleotides and their conjugates. Nucl. Acids Res. 21:5337-5344.

Gait, M.J., Singh, M., and Sheppard, R.C. 1980. Rapid synthesis of oligodeoxyribonucleotides IV. Improved solid phase synthesis of oligodeoxyribonucleotides through phosphotriester intermediates. Nucl. Acids Res. 8:1081-1097.

Gausepohl, H., Pieles, U., and Frank, R.W. 1992. Schiff's base analog formation during in situ activation by HBTU and TBTU. In Peptides: Chemistry and Biology (J.A. Smith and J.E. Rivier, eds.) pp. 523-524. ESCOM, Leiden.

Horn, M. and Novak, C. 1987. A monitoring and control chemistry for solid-phase peptide synthesis. Am. Biotechnol. Lab. 5:12-21.

Koster, H., Stumpe, A., and Wolter, A. 1983. Polymer support oligonucleotide synthesis 13: Rapid and efficient synthesis of oligodeoxynucleotides on porous-glass support using triester approach. Tetrahedron Lett. 24:747-750.

Kumar, P., Ghosh, N.N., Sadana, K.L., Garg, B.S., and Gupta, K.C. 1993. Improved methods for $3 '-O$-succinylation of 2 -deoxyribo- and ribonucleosides and their covalent anchoring on polymer supports for oligonucleotide synthesis. $\mathrm{Nu}$ cleos. Nucleot. 12:565-584.

Kumar, P., Sharma, A.K., Sharma, P., Garg, B.S., and Gupta, K.C. 1996. Express protocol for functionalization of polymer supports for oligonucleotide synthesis. Nucleos. Nucleot. 15:879888 .

Markiewicz, W.T., Groger, G., Rosch, R., Zebrowska, A., Markiewicz, M., Klotz, M., Hinz, M., Godzina, P., and Seliger, H. 1997. A new method of synthesis of fluorescently labelled oligonucleotides and their application in DNA sequencing. Nucl. Acids Res. 25:3672-3680.

Matteucci, M.D. and Caruthers, M.H. 1981. Synthesis of deoxyoligonucleotides on a polymer support. J. Am. Chem. Soc. 103:3185-3191.

Miyoshi, K.-I., Miyake, T., Hozumi, T., and Itakura, K. 1980. Solid-phase synthesis of polynucleotides. II. Synthesis of polythymidylic acids by the block coupling phosphotriester method. Nucl. Acids Res. 8:5473-5489.

Montserrat, F.X., Grandas, A., and Pedroso, E. 1993. Predictable and reproducible yields in the anchoring of DMT-nucleoside-succinates to highly loaded aminoalkyl-polystyrene. Nucleos. $\mathrm{Nu}$ cleot. 12:967-971.

Ogilvie, K.K. and Kroeker, K. 1971. Synthesis of oligothymidylates on an insoluble polymer support. Can. J. Chem. 50:1211-1215. 
Pon, R.T. 1993. Solid-phase supports for oligonucleotide synthesis. In Protocols for Oligonucleotides and Analogs (S. Agrawal, ed.) pp. 465496. Humana Press, Totowa, N.J.

Pon, R.T. and Yu, S. 1997a. Hydroquinone- $O, O^{\prime}$ diacetic acid ('Q-linker') as a replacement for succinyl and oxalyl linker arms in solid phase oligonucleotide synthesis. Nucl. Acids Res. 25:3629-3635.

Pon, R.T. and Yu, S. 1997b. Rapid automated derivatization of solid-phase supports for oligonucleotide synthesis using uronium or phosphonium coupling reagents. Tetrahedron Lett. 38:3331-3334.

Pon, R.T., Usman, N., and Ogilvie, K.K. 1988. Derivatization of controlled pore glass beads for solid phase oligonucleotide synthesis. Biotechniques 6:768-775.

Pon, R.T., Yu, S., Guo, Z., Yang, X., and Sanghvi, Y.S. 1999. Reusable solid-phase supports for oligonucleotide synthesis using hydroquinone$O, O^{\prime}$-diacetic acid (Q-Linker). Nucleos. $\mathrm{Nu}$ cleot. In press.

Pon, R.T., Yu, S., Guo, Z., and Sanghvi, Y.S. 1999. Multiple oligodeoxyribonucleotide syntheses on a reusable solid-phase CPG support via the hydroquinone- $O, O^{\prime}$-diacetic acid $(Q$-Linker) linker arm. Nucleic Acids Res. 27:1531-1538.
Reddy, M.P. and Voelker, P.J. 1988. Novel method for monitoring the coupling efficiency in solid phase peptide synthesis. Int. J. Pept. Protein Res. 31:345-348.

Reddy, M.P., Rampal, J.B., and Beaucage, S.L. 1987. An efficient procedure for the solid phase tritylation of nucleosides and nucleotides. Tetrahedron Lett. 28:23-26.

Sharma, P., Sharma, A.K., Malhotra, V.P., and Gupta, K.C. 1992. One pot general method for the derivatization of polymer support for oligonucleotide synthesis. Nucl. Acids Res. 20:4100-4100.

Story, S.C. and Aldrich, J.V. 1994. Side-product formation during cyclization with HBTU on a solid support. Int. J. Pept. Protein Res. 43:292296.

Walsh, A.J., Clark, G.C., and Fraser, W. 1997. A direct and efficient method for derivatisation of solid supports for oligonucleotide synthesis. Tetrahedron Lett. 38:1651-1654.

Yip, K.F. and Tsou, K.C. 1971. A new polymer support method for the synthesis of ribooligonucleotide. J. Am. Chem. Soc. 93:3272-3276.

Contributed by Richard T. Pon

University of Calgary

Calgary, Alberta, Canada
Synthesis of Unmodified Oligonucleotides 